\title{
PAPER
}

\section{Improving endoscopic resolution and sampling: fluorescence techniques}

\section{Ell}

Gastrointestinal tumours are usually associated with a poor prognosis, as most are only diagnosed at an advanced stage. Every effort to improve intraluminal diagnosis in gastroenterology must therefore be aimed at diagnosing gastrointestinal tract tumours at the earliest stage possible.

mproved training for physicians conducting endoscopy is a fundamental element in the early detection of gatrointestinal tumours. Familiarity with the adenoma-carcinoma sequence in colorectal carcinoma, for example, or the metaplasia-dysplasia-carcinoma sequence in adenocarcinoma of the distal oesophagus, leads to a more consistent examination technique. ${ }^{1-4}$ Practical training in endoscopy has now reached a high standard throughout the world, although it should by no means be regarded as already representing the ultimate that can be achieved.

On the other hand, the quality of the endoscopes used is vital for early recognition of gastrointestinal tumours. Although the introduction of video endoscopy some 25 years ago was regarded as the peak of endoscope development, a further quantum leap has been achieved in image resolution in the past 5 to 10 years, with the introduction of high resolution (megapixel) and magnification endoscopy.

Supplementary procedures such as chromoendoscopy and fluorescence techniques (FT) can further contribute to optimal diagnosis, helping to diagnose not only early carcinomas but also preliminary stages such as high grade intraepithelial neoplasia (formerly known as "severe dysplasia").

The first promising reports on FT were already published more than 10 years ago. ${ }^{5}$ Interest primarily focused on fluorescence spectroscopic techniques. From the point of view of clinical gastroenterologists, however, the vital requirement was to obtain a real time endoscopic image_-not receiving point by point fluorescence measurement, but rather recognising differences in fluorescence directly on the monitor when viewing extensive areas of tissue. Among fluorescence imaging methods, there is currently competition among techniques that use endogenous fluorophores (autofluorescence) and those that use exogenous fluorophores (known as photodynamic diagnosis, PDD) to stimulate fluorescence. The aim in all these efforts is to achieve fluorescence optically guided biopsy sampling of suspicious areas. This is of clinical significance above all in long segment Barrett's oesophagus, and for screening in patients with long term chronic inflammatory bowel diseases. Random biopsy sampling is currently still the standard procedure here.

\section{PRINCIPLES OF FLUORESCENCE DIAGNOSIS}

The principle of fluorescence diagnosis is based on the interaction between light at a specific wavelength and fluorophores in tissue. Light sources that can be used include incoherent light sources (for example, xenon) or monochromatic laser light. When light penetrates tissue, it causes not only reflection and absorption, but also fluorescence. Depending on the fluorophore used, only light at a specific wavelength is maximally absorbed, and in turn variably emitted. As a result of the stimulation process, blue stimulation light, for example, loses some of its energy and is therefore given off as longer wavelength red fluorescence. "Colour differences" between normal and neoplastic tissue arise here as a result of endogenous and exogenous fluorophores.

\section{Autofluorescence}

Autofluorescence is based on the stimulation of endogenous fluorophores, particularly the COenzymes of the respiratory chain $(\mathrm{NADH}$, collagen, flavin, etc). As their excitation and emission bands are quite broad and often show spectral overlapping, it is still difficult to distinguish between these fluorophores. It is only when a suitable stimulation wavelength is selected that the individual fluorophores causing autofluorescence can be distinguished. A number of different pathological processes-mainly neoplasias, but also inflammation and ischaemia-affect the metabolic, oxidative condition of the cells and thus their autofluorescence. In addition, the thickness of the mucosa or epithelial layer can change the autofluorescence. After stimulation with ultraviolet or short wavelength light, these fluorophores emit longer wavelength light. Thickening of the mucosa produces a weakened green autofluorescence in the collagen and elastin contained in the submucosa. The predominantly green fluorescence $(450-550 \mathrm{~nm})$ in the submucosa (collagen) is masked by dysplastic/ malignantly transformed mucosa, the red fluorescence of which (600-700 nm) then predominates. The main advantage of the autofluorescence technique is the fact that it is not necessary to administer a chemical substance to the patient before fluorescence can be detected. Administering a drug has considerable medical and also legal implications, quite apart from the tremendous 
costs involved in the process of obtaining registration and approval for an exogenous fluorophore as a medication. However, the faintness of endogenous fluorophores, the complexity of the interactions between the numerous endogenous pigments that are present, and the varied effects of different tissue qualities on autofluorescence behaviour, all make sensitive and sophisticated detection systems necessary to obtain meaningful information. ${ }^{6-12}$

\section{Exogenous fluorescence}

In contrast with autofluorescence, various exogenous photosensitisers-classically porphyrins-are used when fluorescence is induced by a drug in the technique known as PDD. These can be applied either for imaging or for spectroscopy alone. The decisive difference between these and endogenous fluorophores is that there are evidently substances that accumulate more, with relative selectivity, in neoplastic cell systems. For diagnostic purposes, 5-aminolevulinic acid (5-ALA) is currently the substance attracting the greatest interest. It is not really a photosensitiser, but rather a precursor in heme biosynthesis. The reduced ferrochelatase activity in neoplastic cells has a decisive role here for selective accumulation in malignant tumours in comparison with normal tissue. Exogenously administered 5-ALA bypasses an inhibitory feedback mechanism in heme biosynthesis and leads to accumulation of protoporphyrin IX (PpIX) in neoplastic tissues. Selective accumulation of 5-ALA or PpIX in neoplastic tissues was initially demonstrated in skin tumours, and later also in numerous other types of organ tumour. However, the use of exogenous substances that produce a stronger and clearer fluorescence signal because of comparatively specific accumulation also has some disadvantages. In addition to the legal and medicolegal aspects already mentioned, administration of 5-ALA is associated with brief but non-negligible photosensitisation of the skin, so that the patient needs to be protected from sunlight for about one day. ${ }^{13-17}$

\section{Fluorescence spectroscopy}

Endoscopic fluorescence spectroscopy procedures usually use lasers as light stimulation sources, with wavelengths between 300 and $480 \mathrm{~nm}$. The light is introduced into the gastrointestinal hollow organs via glass fibres under endoscopic vision. The fluorescence light can return via the same fibres, or via separate light conductors. A fluorescence spectrometer detects the wavelength dependent signals. Background subtraction techniques and ratio calculations for two or more wavelengths are usually used. Particularly when only autofluorescence is being used, sophisticated algorithms are needed to calculate the spectral contributions of different fluorophores to derive a value that indicates the presence or absence of neoplasia in the tissue area being investigated. One evident drawback of spectroscopy is the fact that only small areas of tissue can be examined. The optical probes used to measure reflectance spectra through an endoscope sample about $0.05-1 \mathrm{~mm}^{2}$ of tissue at each site. The total amount of tissue examined spectroscopically, even with multiple measurements, is thus very small compared with fluorescence imaging, and it is possible that regions of neoplastic tissue may be missed because of sampling errors. In addition, the endoscopist needs to detect suspicious areas first before the probe can be used for fluorescence spectroscopy. ${ }^{5} 6^{10-12} 1718$

\section{Fluorescence imaging}

From the clinical viewpoint, a real time endoscopic fluorescence imaging system is of course highly attractive, as this is the only way of achieving complete fluorescence diagnosis of large, continuous areas of tissue. However, the method has low sensitivity and specificity rates, and these deficiencies have to be compensated for by using special cameras with image intensifiers, or time integration facilities. Two commercial systems are currently available:

- The Xillix Laser-Induced Fluorescence Endoscopy Gastrointestinal (LIFE-GI) system (Xillix Technologies, Vancouver, Canada) is based on capturing autofluorescence signals stimulated with blue light at a wavelength of $437 \mathrm{~nm}$. Using fibrescopes, two image intensifier cameras attached to the endoscope outside the body are used to capture autofluorescence signals in the green and red spectral ranges, which are fused to create a real time "red-green" image. Generally, there is less green fluorescence in neoplastic tissue than in normal tissue, while red fluorescence is stronger in neoplastic tissue than in normal tissue. The unwieldy clinical operating characteristics of this system have in the meantime been substantially improved in a second version (LIFE II). However, the new system still only uses fibre endoscopes, and not video endoscopes. ${ }^{89} 1920$

- The second real time fluorescence system is based on the use of exogenous fluorophores, and detects 5-ALAinduced PpIX fluorescence. The process was initially developed by the Storz company in Tuttlingen, Germany, and was known as D-Light; in the meantime a basically similar system is also available from the R Wolf company in Knittlingen, Germany (Combilight PDD). For 5-ALA fluorescence endoscopy, the set up is illustrated in figure 1. The excitation light is a short arc xenon lamp with a specially designed yellow short pass filter $(440 \mathrm{~nm})$. Blue and conventional white light are delivered to the organ segments via the illumination bundle of a standard fibrescope at $200 \mathrm{~mW}$. The fluorescence light is collected by the endoscope's imaging bundle. An observation filter (long pass, $450 \mathrm{~nm}$, Schott, Germany) is mounted on the eyepiece of the endoscope. The light is designed to transmit not only tissue autofluorescence and PpIX fluorescence, but also part of the excitation light, for better orientation during the examination. For documentation, a video camera with target integration is attached to the flexible fibreoptic endoscope. 5-ALA is usually supplied as hydrochloride $98 \%$ pure powder. The patients receive a solution of ALA in mineral water (dose $10 \mathrm{mg} / \mathrm{kg}$ body weight) orally four to eight hours before fluorescence endoscopy. After ingesting the ALA, the patients are kept in slightly darkened rooms for 36 hours. Sensitisation with ALA, which is transformed to PpIX in neoplastic tissue in higher concentrations than in non-malignant tissue, leads to a characteristic red fluorescence on exposure to blue light, as PpIX has a strong absorption band in the violet spectral range $(380-420 \mathrm{~nm})$. The red fluorescence emitted presents two bands at 635 and $700 \mathrm{~nm} \cdot{ }^{13-16}$

\section{Clinical experience in gastrointestinal endoscopy}

Most experimental ex vivo studies and clinical experimental studies have been carried out using fluorescence spectroscopy. ${ }^{51117}$ Various spectral and fluorescence imaging systems have been used to analyse the fluorescence data. The results have shown sometimes substantial differences between in vitro and in vivo conditions. In individual human malignant tumours, autofluorescence emission spectra show peaks in the $630-690 \mathrm{~nm}$ wavelength range. ${ }^{18}$ In ex vivo studies of oesophageal carcinomas (adenocarcinoma), high levels of fluorescence activity were observed in laser induced autofluorescence (stimulation wavelength $405 \mathrm{~nm}$ ) in normal tissue at $500 \mathrm{~nm}$, with smaller peaks for normal tissue, dysplastic tissue and malignant tissue at $630 \mathrm{~nm}$ and 700 $\mathrm{nm} .{ }^{17}$ When a ratio is calculated between the fluorescence activity at $500 \mathrm{~nm}$ and $630 \mathrm{~nm}$, highly significant differences are found between malignant and benign Barrett's mucosa. ${ }^{17}$ Recent in vivo studies in oesophageal and gastric tumours show specific changes in the autofluorescence spectra of 


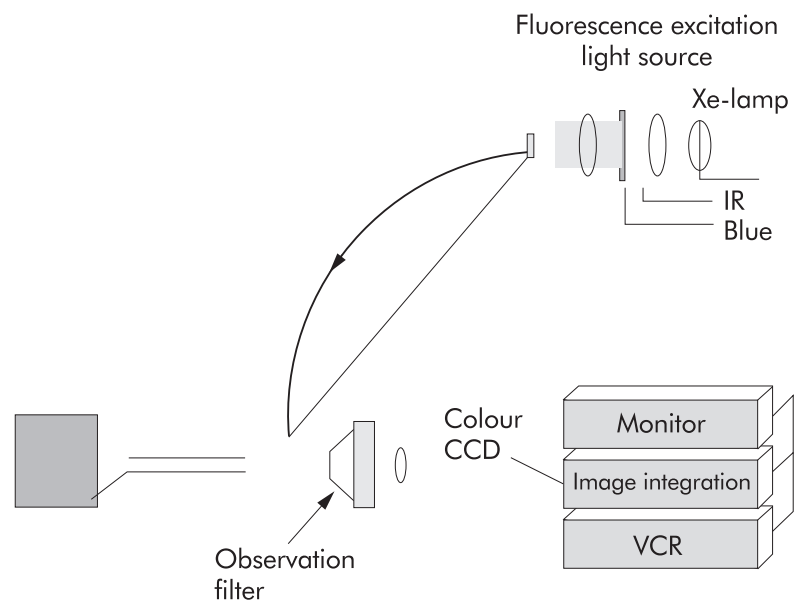

Figure 1 Schematic diagram of the photodynamic diagnosis system.

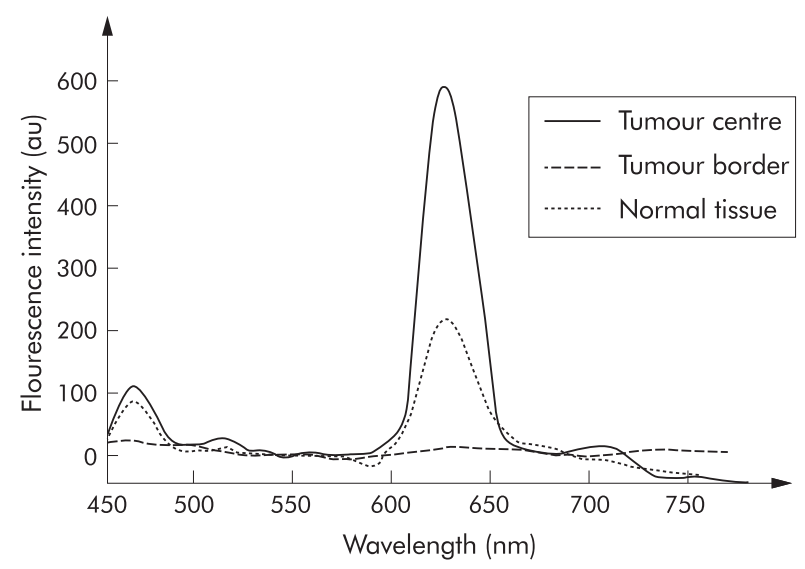

Figure 2 Endoscopic fluorescence spectra from high grade dysplasia (solid line) and normal tissue (dotted line) in Barrett's oesophagus. Normal tissue shows only weak protoporphyrin IX fluorescence.

malignant tumours in comparison with the corresponding normal tissue. ${ }^{12}$ Using complex mathematical algorithms, a sensitivity of $97 \%$ and a specificity of $95 \%$ can be calculated for the autofluorescence technique. Autofluorescence measurements in the colon have shown that there are reduced fluorescence intensities in adenomatous neoplasia, in comparison with normal colonic mucosa, in the spectral range 500-550 $\mathrm{nm}$. To improve tissue identification using autofluorescence in the colon, excitation-emission matrices (EEMs) have been recorded with excitation wavelengths from $250 \mathrm{~nm}$ to $500 \mathrm{~nm}$ in steps of $10 \mathrm{~nm}$, with corresponding fluorescence emission up to $700 \mathrm{~nm}$. Using 5-ALA as an exogenous fluorophore, it was possible to distinguish clearly between tumours in Barrett's oesophagus and Barrett's metaplasia, with the typical PpIX peaks at $635 \mathrm{~nm}$ and $705 \mathrm{~nm} .{ }^{13}$ (Figure 2).

A review of the available literature reports shows that in the early 1990s, the published studies almost exclusively used fluorescence spectroscopy based on laser induced autofluorescence. Initially, these were mainly experimental and ex vivo studies, but in recent years in vivo studies have also appeared, some of which have also used exogenous fluorophores. More than half of the published studies have focused on the large bowel, followed by studies in the oesophagus.

With the development of the fluorescence imaging system, scientific interest concentrated more on the oesophagus-not least because of the increasing clinical significance of Barrett's carcinoma, as well as a recognition that fluorescence procedures for detecting or differentiating between colonic polyps were too elaborate or were not genuinely clinical relevant. Early colonic neoplasias can be recognised much better using high resolution endoscopy, with or without chromoendoscopy, than was possible 10 or 20 years ago. By contrast, intraepithelial neoplasias (high grade, low grade) in Barrett's oesophagus cannot usually be recognised, even using the above methods, so that there is a genuine and significant need for diagnostic improvement here. This is underlined by the fact that new local endoscopic treatment options as photodynamic therapy and mucosal resection are available now. ${ }^{21}{ }^{22}$ The same applies to the detection of dysplasias in patients with chronic inflammatory bowel diseases, in whom conventional diagnostic procedures have limitations. A third diagnostic problem situation is early recognition of gastric carcinomas. In contrast with sporadic colorectal carcinoma, conventional endoscopy is not capable of achieving early recognition of the preliminary stages of cancer here.

The new fluorescence imaging systems allow both conventional "white light" endoscopy and fluorescence endoscopy in one session. In addition, they permit full inspection of the area at risk-for example, the large bowel. This is an advance over point by point measurements in which the measurement process is random, equivalent to a random biopsy procedure, unless the endoscopist has already recognised a lesion, so that targeted fluorescence measurement can be carried out, equivalent to a "virtual biopsy," with a subsequent "optically guided biopsy."

There have few reports on the use of the LIFE-GI autofluorescence system, with publications mainly being concerned with ex vivo studies or case reports on clinical experience. ${ }^{8} 9192023$ In an ex vivo study, a group of Japanese authors have pointed out that the LIFE-GI system is probably unsuitable for detecting the undifferentiated type of gastric carcinoma, as the detection rate for these lesions was significantly poorer than for the differentiated type. ${ }^{9}$ In an initial small series, Haringsma et al found that the LIFE-GI system was slightly superior to random biopsy in Barrett's oesophagus. This was confirmed in a comparable series. ${ }^{20}$ However, on the basis of personal communications from researchers using the LIFE-GI method experimentally in everyday clinical work, the system seems to be extremely elaborate and rather unpractical. The fluorescence unit as a whole is extremely expensive because of the use of a laser system, and this is a further limitation on its clinical use. The later prototype, LIFEII, has been substantially improved, ${ }^{19}$ and a research group in the Netherlands will be reporting on the diagnostic yield in the near future. ${ }^{24}$

In contrast with the LIFE-GI system, fluorescence imaging systems based on 5-ALA use xenon lamps as the stimulation light source, instead of lasers. The system as a whole is easy to handle and reasonably priced. On the other hand, as mentioned earlier, the costs and effort associated with 5-ALA are not negligible. In addition to case reports, a series of 47 patients with Barrett's oesophagus in whom the technique was used was published last year. ${ }^{15}$ Only 10 of the 47 showed dysplasia, and previously unidentified dysplasias were found using the D-Light system in three of the patients. Our own research group is reporting this year on 43 patients with high grade neoplasia and early Barrett's cancer who were examined using random biopsy under white light vision and also with 5-ALA-induced fluorescence endoscopy. ${ }^{13}$ The patients were studied twice-with conventional endoscopy and four quadrant biopsies in the first series (group I), and with fluorescence detection (photodynamic diagnosis) in the second series (group II). Firstly, four quadrant random biopsy specimens every $2 \mathrm{~cm}$ were taken during routine video endoscopy (Fujinon 450 HR, Fujinon, Willich, Germany). In the second series, two to eight days later, PDD was performed three hours after oral sensitisation with ALA. Biopsies were taken of areas with positive red fluorescent tissue (one to four 
specimens per lesion) and of non-fluorescent areas (two specimens per patient). In group I, a total of 448 quadrant specimens were sampled, while in group II, 175 samples were taken. Overall, the PDD technique led to a significantly lower total number of biopsy specimens being obtained per patient compared with random biopsies $(\mathrm{n}=448 v 175, \mathrm{p}<0.0001)$. In addition, the average number of specimens taken with PDD was much lower than with random biopsies ( $4 v 9, \mathrm{p}<0.0001)$. In relation to the length of the Barrett's segment, the difference was significant only in patients with long segment Barrett's oesophagus $(p<0.0001$. PDD led to a significantly higher proportion of biopsy specimens containing dysplasia or early cancer despite the smaller number of samples obtained per patient. Significant differences between the two techniques in the detection of HGD or early cancer were present in both short segment and long segment Barrett's oesophagus. The sensitivity was $81.7 \%$ for PDD, while a sensitivity rate of $25.9 \%(p<0.001)$ was found using random biopsy in the study. No dysplasia, early cancer, or inflammation was detected in fluorescence negative areas of Barrett's mucosa, and consequently no false negative fluorescence findings were obtained. False positive fluorescence was associated either with microscopic inflammation of the mucosa or with low grade dysplasia. Although these results are still preliminary in nature and need to be confirmed by other research groups, they suggest that ALA induced fluorescence diagnosis can be reliably used for recognition of discrete mucosal abnormalities such as high grade dysplasia and early cancer in Barrett's oesophagus. Open questions that remain undoubtedly include the optimal dose of 5-ALA and whether topical application is capable of being as effective as oral administration. ${ }^{13} 15$

The results of one pilot study conducted in Germany on 5-ALA fluorescence detection in the colon has now also been published. ${ }^{16}$ 5-ALA fluorescence detection was found to be equivalent to whitelight endoscopy for detecting neoplasias. As mentioned earlier, the use of fluorescence for screening in patients with chronic inflammatory bowel diseases is of much greater interest than for detecting neoplasia in the inflammation free colon. The preliminary data reported by the group in Regensburg, Germany, are very promising here (H Messmann, personal communication). However, the limitations of in vivo fluorescence diagnosis in the large bowel include the problems of fecal contamination and the high level of autofluorescence of fecal residues. On the other hand, any sort of inflammatory activity in the mucosa also produces difficult to interpret fluorescence effects. In my view, the use of fluorescence endoscopy in the large bowel will in the future continue to be useful and feasible mainly for scientific purposes, because of the limited range of indications and the substantial obstructive factors present. Widespread clinical use is unlikely in my opinion. By contrast, increased use of the technique to detect neoplastic lesions in the stomach could lead to it becoming an established method, as the obstructive factors mentioned above are not present there. ${ }^{93}$

To sum up, the clinical indications for fluorescence endoscopy will in the future be limited mainly to patients with Barrett's oesophagus. Furthermore, possible indications could be the screening of patients with long term ulcerative colitis and chronic inflammatory bowel diseases, and perhaps also patients who are at increased risk for gastric carcinoma. Spectroscopic procedures still have higher sensitivity and specificity rates for identifying neoplastic lesions than imaging fluorescence systems. On the other hand, they are more elaborate and only permit point by point fluorescence measurements. From the clinical point of view, only real time endoscopic fluorescence imaging systems represent a practicable solution. The systems that are currently available still have weaknesses, and will need to undergo thorough clinical evaluation once they have been technically optimised. In comparison with incoherent light sources, laser based methods seem to be too elaborate and expensive, and the former are likely to replace them. In addition, fluorescence detection will have to be possible in the future using high resolution video endoscopes. Continued use of fluorescence endoscopy only on the basis of the fibrescopes so far available will soon become anachronistic. Ultimately, whether and to what extent fluorescence endoscopy will find its place in clinical gastroenterology will only be decided in comparison with the other imaging options available (high resolution endoscopy, magnification endoscopy, chromoendoscopy, etc).

\section{REFERENCES}

1 Lagergren J, Bergström R, Lindgren A, et al. Symptomatic gastroesophageal reflux as a risk factor for esophageal adenocarcinoma. N Engl J Med 1999;340:825-31.

2 Prach AT, MacDonald TA, Hopwood DA, et al. Increasing incidence of Barrett's oesophagus: education, enthusiasm, or epidemiology? Lancet 1997;350:933.

3 Hameeteman W, Tytgat GNJ, Houthoff HJ, et al. Barrett's esophagus: development of dysplasia and adenocarcinoma. Gastroenterology 1989;96:1249-56.

4 Blot WJ, Devesa SS, Kneller RW, et al. Rising incidence of adenocarcinoma of the esophagus and gastric cardia. JAMA 1991;265:1287-9.

5 Schomaker KT, Frisoli JK, Compton CC, et al. Ultraviolet laser-induced fluorescence of colonic polyps. Gastroenterology 1992;102:1 155-60.

6 Cothren RM, Richards-Kortum R, Sivak MV, et al. Gastrointestinal tissue diagnosis by laser-induced fluorescence spectroscopy at endoscopy. Gastrointest Endosc 1990;36:105-11.

7 Stepp H, Sroka R, Baumgartner R. Fluoerescence endoscopy of gastrointestinal diseases: Basic principles, techniques and clinical experiences. Endoscopy 1998;30:379-86.

8 Abe S, Izuishi K, Tajiri H, et al. Correlation of in vitro autofluorescence endoscopy images with histopathologic findings in stomach cancer. Endoscopy 2000;32:281-6.

9 Haringsma J, Prawirodirido W, Tytgat GNJ. Accuracy of fluorescence imaging of dysplasia in Barrett's esophagus. Gastroenterology 1999:116:A 418

10 Bourg-Heckly G, Blais J, Padilla J-J, et al. Endoscopic ultraviolet-induced autofluorescence spectroscopy of the esophagus: tissue characterization and potential for early cancer diganosis. Endoscopy 2000;32:756-65.

11 Panjehpour M Overholt BF, Vo-Dinh T, et al. Endoscopic fluorescence detection in high-grade dysplasia in Barrettt's esophagus. Gastroenterology 1996;111:93-101.

12 Mayinger B, Horner $P$, Jordan $M$, et al. Light-induced autofluorescence spectroscopy for the endoscopic detection of esophageal cancer. Gastrointest Endosc 2001;54:195-201

13 Gossner L, Stepp H, Ell C, et al. Photodynamic diagnosis of high-grade dysplasia and early cancer in in Barrett's esophagus using 5-aminolevulinic acid. Am J Gastroenterol (in press).

14 Messmann H, Knüchel R, Bäumler W, et al. Endoscopic fluorescence detection of dysplasia in patients with Barrett's esophagus, ulcerative colitis, or adenomatous polyps after 5-aminolevlinic acid-induced protoporphyrin IX sensitation. Gastrointest Endosc 1999;49:97-101.

15 Endlicher E, Knuechel R, Hauser T, et al. Endoscopic fluorescence detection of low and high grade dysplasia in Barrett's oesophagus using systemic or local 5-aminlevulinic acid sensitization. Gut 2001;48:314-19.

16 Brand S, Stepp H, Sackmann M, et al. Detection of colonic dysplasia by light-induced fluorescence endoscopy: a pilot study. Int J Colorect Dis 1999; 14:63-8

17 Stael von Holstein C, Nilsson AMK, Andersson-Engels S, et al. Detection of adenocarcinoma in Barrett's oesophagus by means of laser induced fluorescence. Gut 1996;39:711-16.

18 Eker C, Montan S, Jaramillo E, et al. Clinical spectral characterisation of colonic mucosal lesions using autofluorsecence and delta aminolevulinic acid sensitisation. Gut 1999:44:511-18.

19 Haringsma J, Tytgat G N J, Hiroyuki Y, et al. Autofluorescence endoscopy: feasibility of detection of $\mathrm{GI}$ neoplasms unapparent to white light endoscopy with an evolving technology. Gastrointest Endosc 2001;53:642-9.

20 DaCosta RS, Wilson BC, Marcon NE. Light induced fluorescence endoscopy of the gastrointestinal tract. Gastointest Endosc Clin N Am 2000; 10:37-69.

21 Gossner L, Stolte M, Ell C, et al. Photodynamic ablation of high-grade dysplasia and early cancer in Barrett's esophagus by means of 5-aminolevulinic acid. Gastroenterology 1998;114:448-55.

22 Ell C, May A, Gossner L, et al. Endoscopic mucosal resection of early cancer and high grade dysplasia in Barrett's esophagus. Gastroenterology 2000; 1 18:670-7.

23 Watanabe H, Ogihara T, Namihisa A, et al. Usefulness of a new autofluorescence endoscopic imaging system for the diagnosis of gastric neoplasms. Endoscopy 1996;28. 